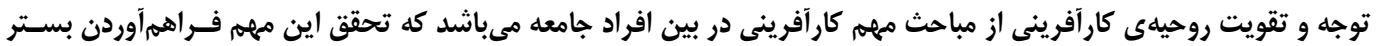

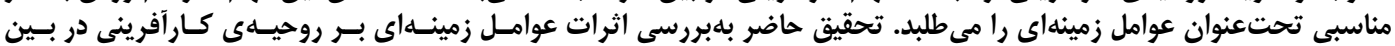

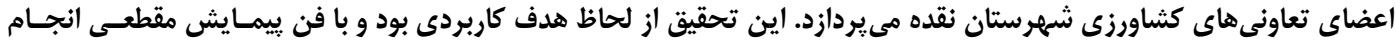

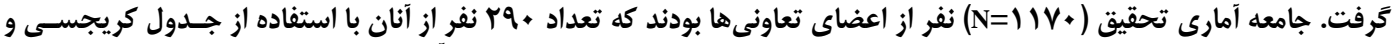

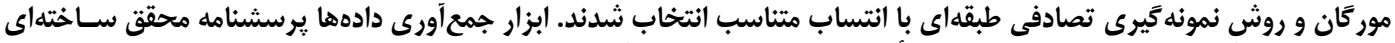

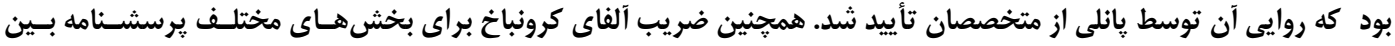

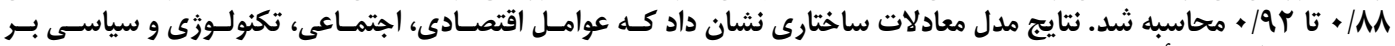

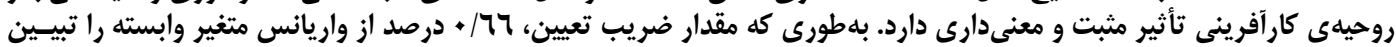

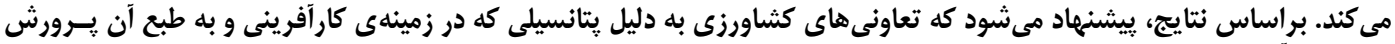

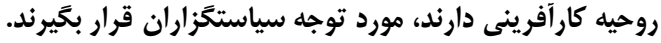

وازههاى كليدى: عوامل زمينهاى، روحيهى كار آفرينى، تعاونىهاى كشاورزى، شهرستان نقده

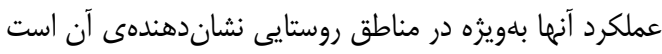

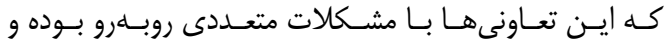

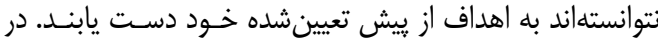

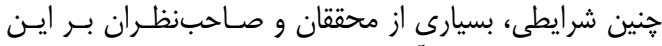

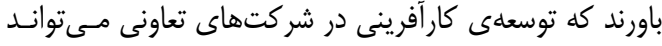

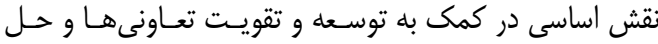

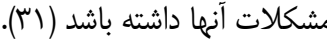

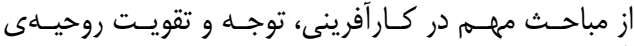

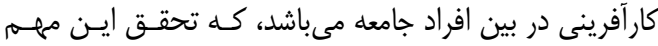

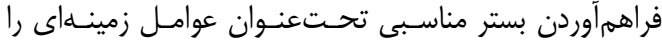

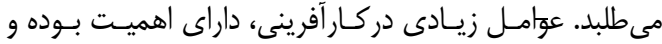

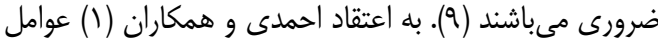

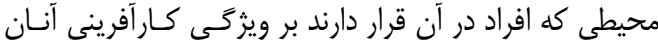

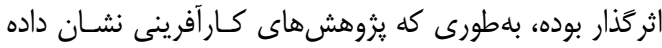

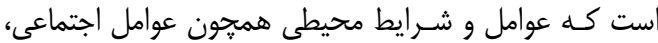

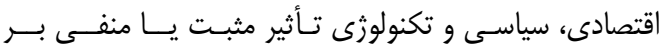

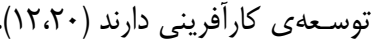

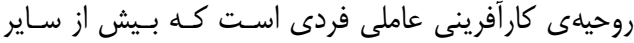

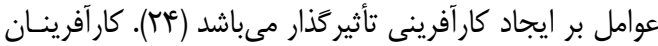

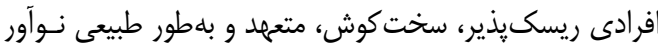

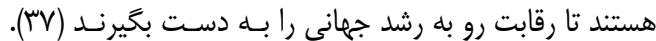

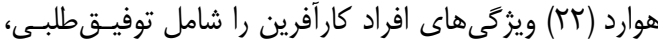

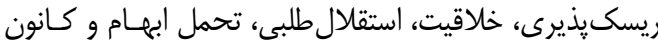

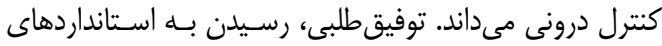

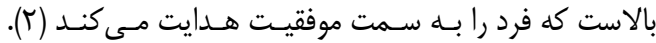

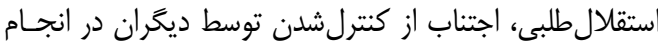

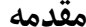

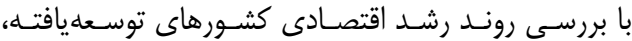

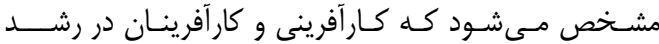

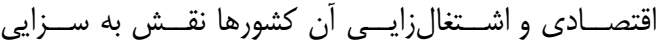

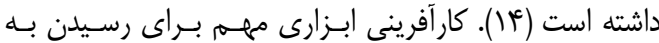

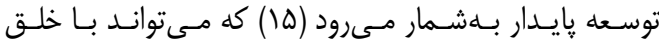

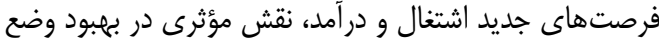

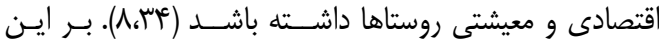

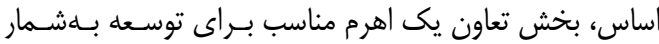

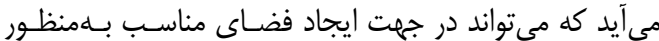

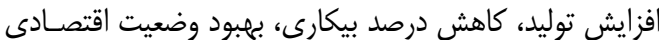

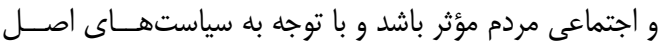

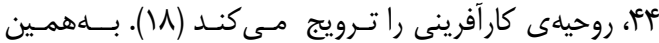

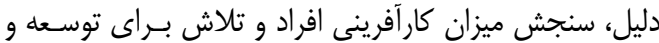

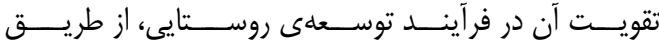

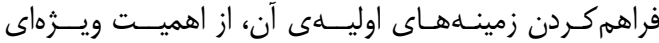

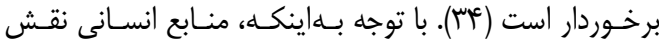

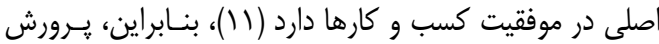

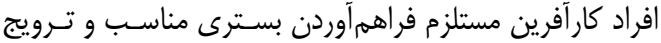

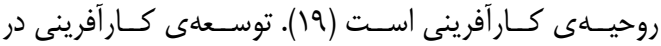

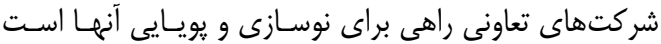

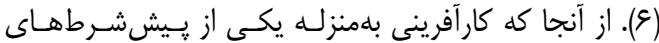

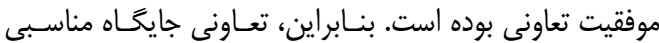

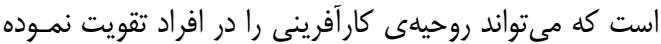

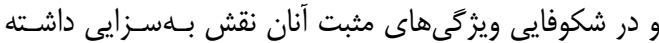

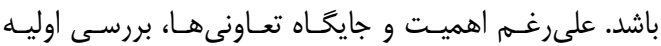




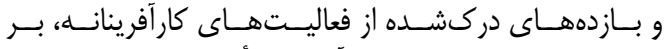

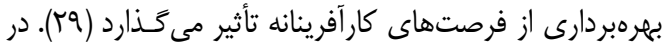

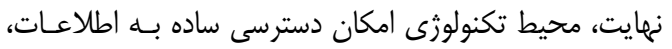

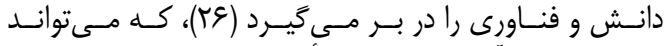

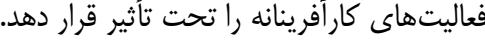

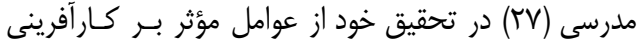

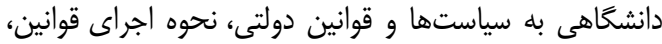

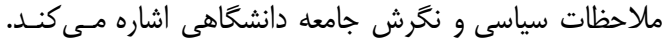

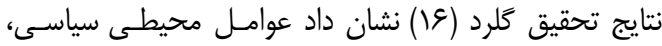

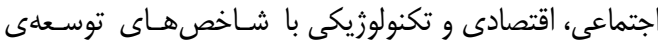

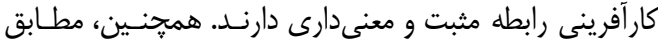

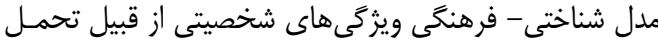

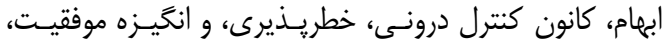

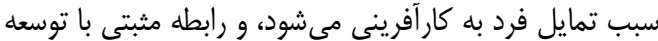

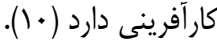

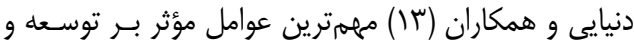

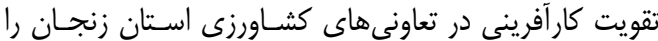

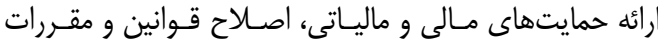

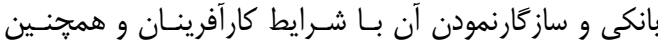

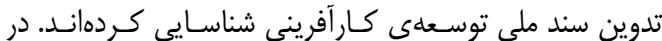

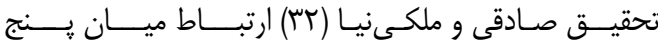

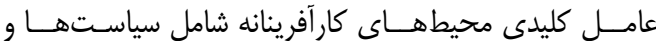

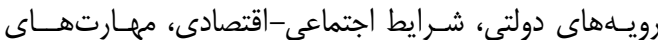

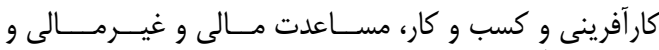

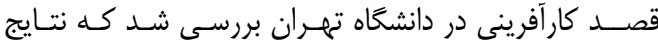

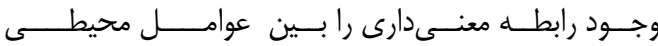

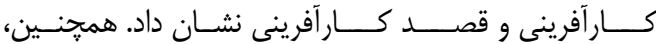

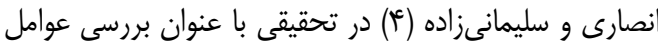

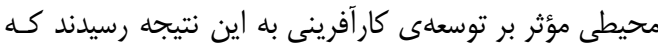

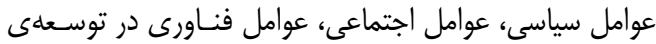
كارأفرينى تأثير دارند.

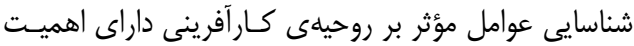

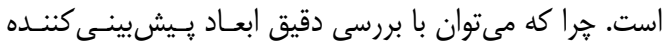

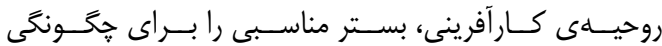

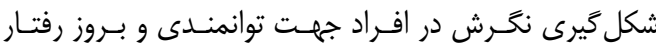

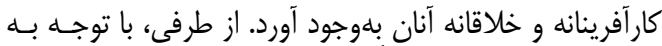

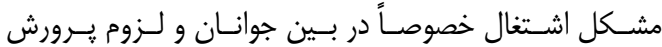

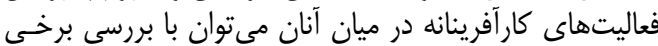

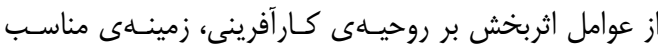

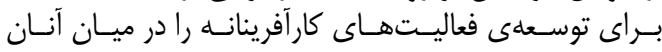

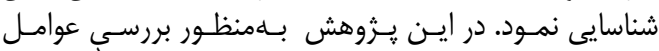

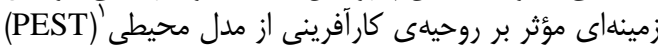

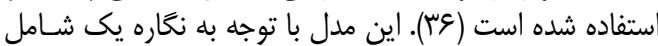

عوامل سياسى، اقتصادى، اجتماعى و تكنولوزيكى مي بـ باشد.

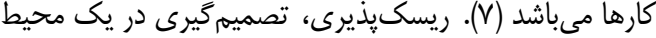

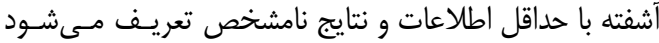

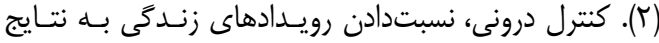

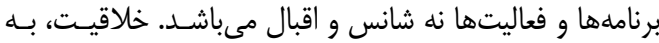

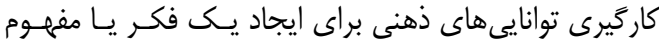

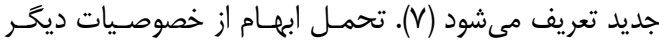

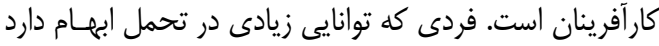

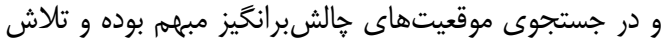

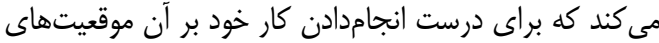

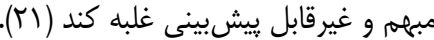

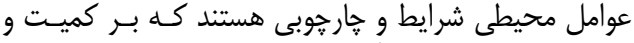

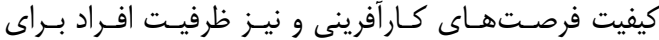

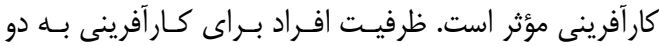

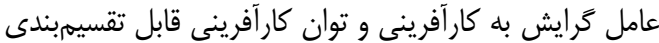

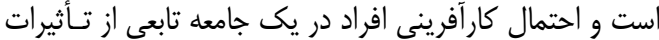

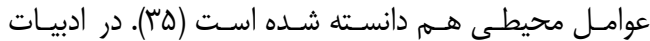
كارآفرينى محيط مجيط شامل كليه عوامل، متغيرها

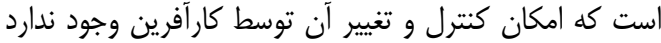

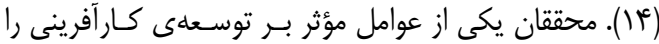

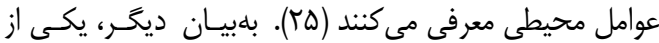

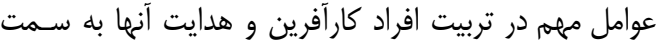

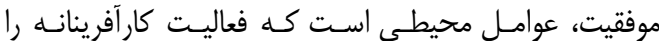

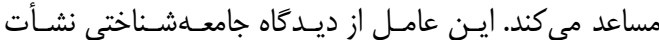

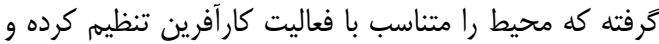

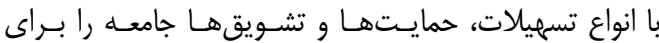

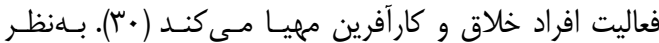

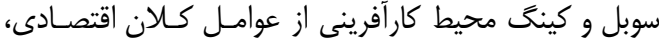

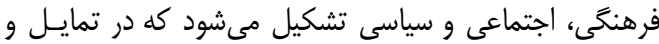

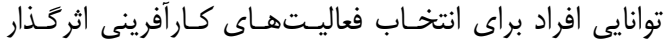

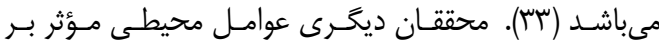

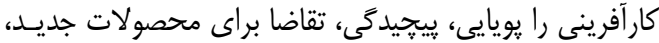

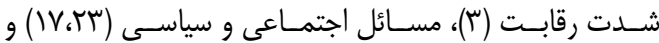

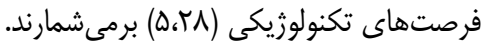

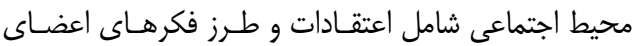

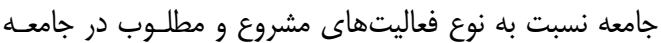

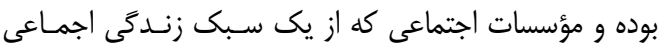

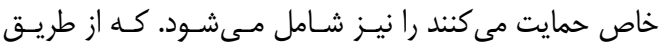

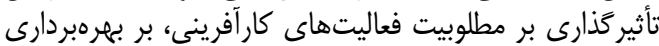

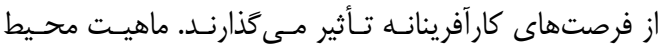

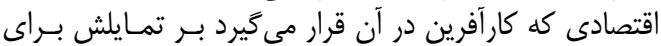

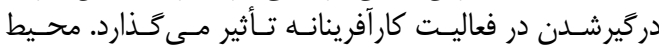

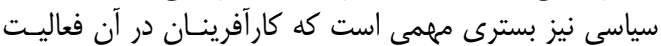

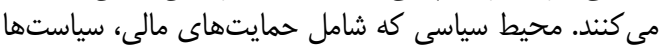

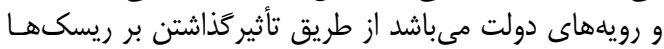




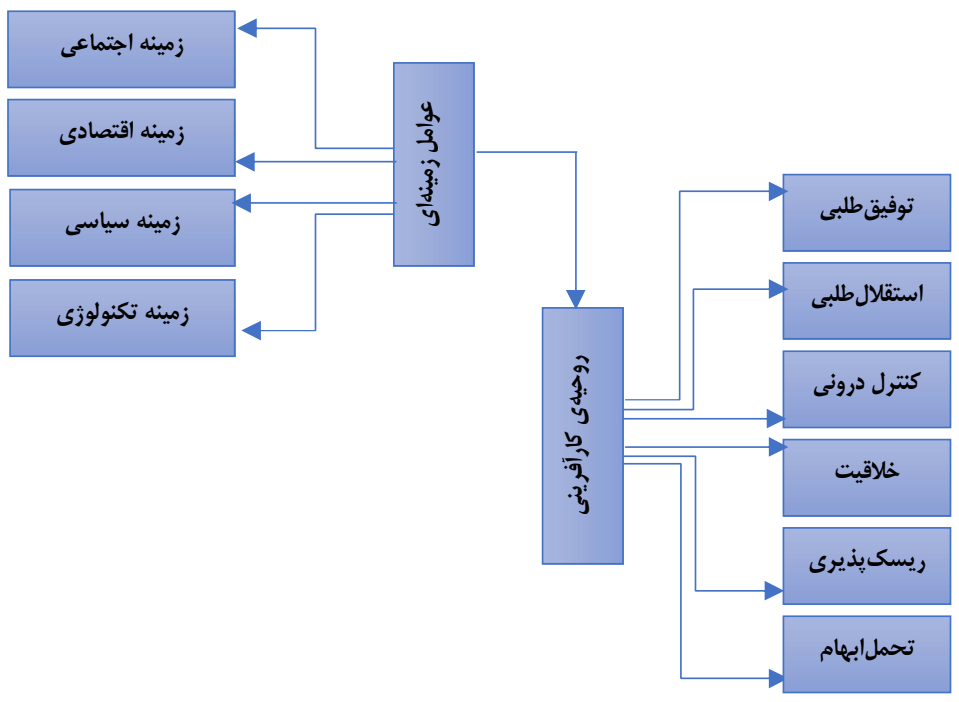

شكل (- خارجوب مفهومى يزوهش (منبع: نتايج حاصل مرور ادبيات)

Figure 1. Conceptual framework of the research

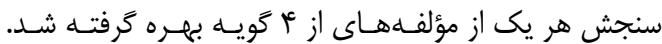

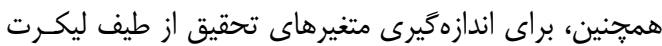

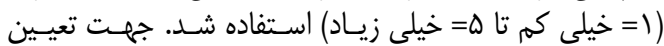

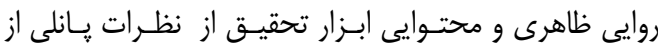

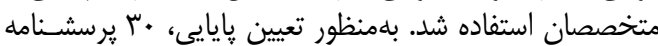

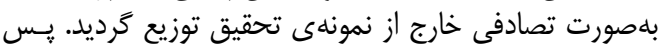

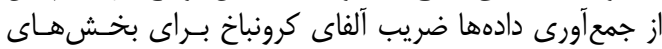

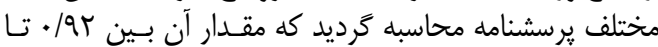

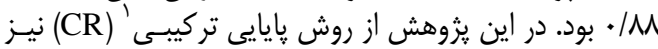

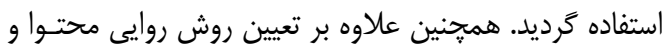

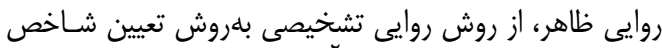

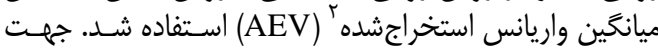

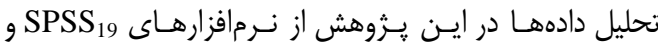
LISREL

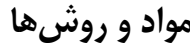

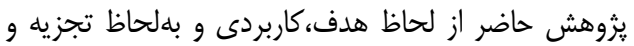

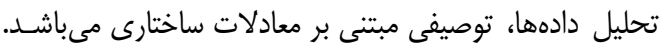

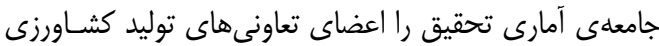

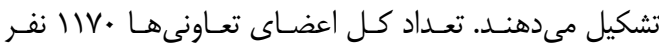

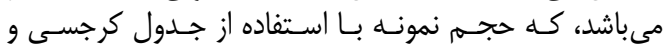

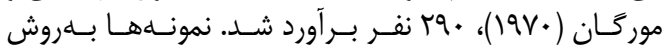

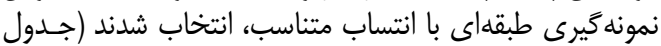

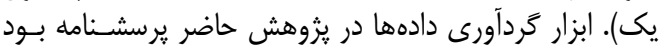

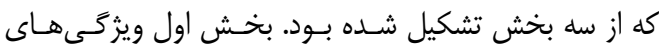

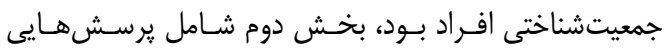

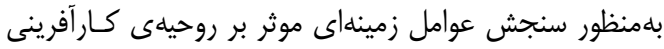

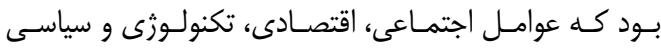

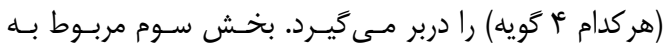

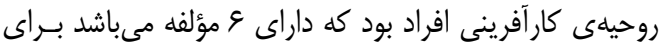

Table 1. Statistical sample and population of agricultural cooperative

جدول (- جامعه و نمونه آمارى اعضاى شركتهاى تعاونى كشاورزى

\begin{tabular}{|c|c|c|c|}
\hline تعداد نمونه & ت تعداد اعضا & شركت تعاونى & رديف \\
\hline $1 q$ & ا 197 عضو & كاوداران نقده & 1 \\
\hline 98 & V V r عضو & دامداران محمديار & r \\
\hline 9 & عسواحد & مرغداران نقده & r \\
\hline ar & Tr TV عضو & مكانيزاسيون & $\Delta$ \\
\hline \multirow[t]{2}{*}{$v^{e}$} & 19 אضو & شركت تعاونى توليد كلوان & $r$ \\
\hline & 118. & & جامعه آمارى \\
\hline rq. & & & نمونه \\
\hline
\end{tabular}




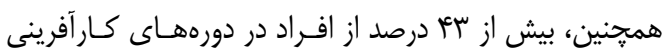

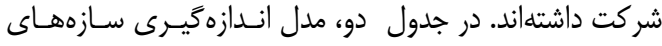

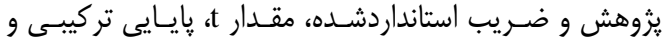

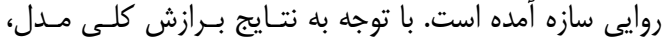

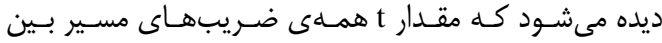

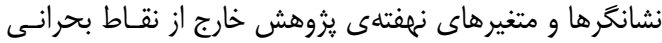

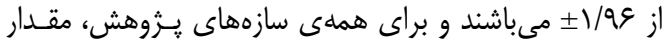

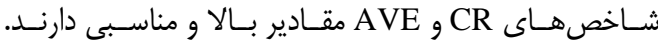

نتايج و بحث

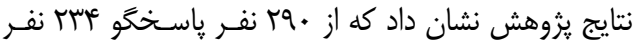

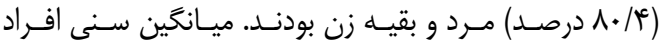

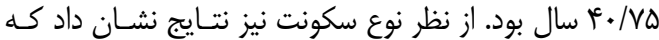

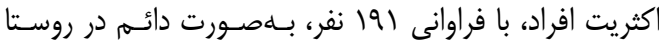

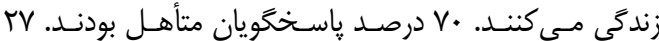

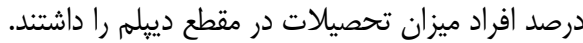

Table 2. Summary of measurement models' latent structures in the research

\begin{tabular}{|c|c|c|c|c|c|c|c|}
\hline AVE & $\mathrm{CR}$ & $\mathrm{T}$ & ضريب استاندارد شده & نوع متغير & 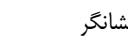 & & سازه \\
\hline \multirow{4}{*}{$\cdot / \Delta \Delta$} & \multirow{4}{*}{ - /AT } & - & $\cdot / 0$ & \multirow{4}{*}{ مستقل } & سياسى & $\mathrm{A}_{1}$ & \multirow{4}{*}{ عوامل زمينهاى (AA) } \\
\hline & & $\mathrm{V} / \overline{8} \mathrm{~V}^{\mathrm{m}}$ &.$/ 94$ & & تكنولوزى & $\mathrm{A}_{2}$ & \\
\hline & & $\Lambda / V^{w^{3 * 3}}$ & $\cdot / \lambda F^{f}$ & & اجتماعى & $\mathrm{A}_{3}$ & \\
\hline & & 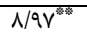 &.$/ 94$ & & اقتصادى & $\mathrm{A}_{4}$ & \\
\hline \multirow{6}{*}{.19} & \multirow{6}{*}{.$/ 9$} & - &.$/ 199$ & \multirow{6}{*}{ وابسته } & توفيقطلبى & $\mathrm{B}_{1}$ & \multirow{6}{*}{ روحيهى كارآفرينى (BB) } \\
\hline & & $\mid r / \Gamma \Lambda^{*}$ & .199 & & استقلال طلبى & $\mathrm{B}_{2}$ & \\
\hline & & $\mid r / T V^{*}$ &.$/ 99$ & & كنترل درونى & $\mathrm{B}_{3}$ & \\
\hline & & $1 f / \Lambda Q^{*}$ & $\cdot / \Lambda$ & & خلاقيت & $\mathrm{B}_{4}$ & \\
\hline & & $\mid f / N 8^{* * 3}$ & $\cdot / \Lambda$ & & ريسكيذيرى & $\mathrm{B}_{5}$ & \\
\hline & & 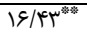 & $\cdot / \mathrm{AV}$ & & تحمل ابهام & $\mathrm{B}_{6}$ & \\
\hline
\end{tabular}

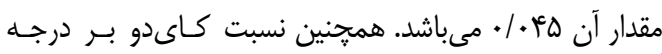

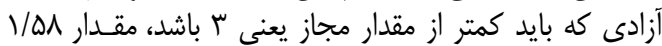
را نشان مى كىهد.

Table 3. Fitness indices

\begin{tabular}{|c|c|c|}
\hline نتايج در يزوهش & 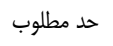 & شاخص برازش \\
\hline.$/ 94$ & 2.19 . & شاخص برازندگى تطبيقى (CFI) \\
\hline.$/ 9$. & 2.19. & شاخص ميزان انطباق (GFI) \\
\hline r & $2 . / 9$. & شاخص نرمشده برازندگى (NFI) \\
\hline.$/ 94$ & 2.19. & شاخص نرمنشده برازندگى (NNFI) \\
\hline.$/ 94$ & 2.19. & شاخص برازندگى فزاينده (IFI) \\
\hline $.1 .+0$ & $\leq \cdot 1 \cdot 1$ & شاخص ريشه دوم برآورد واريانس خطاى تقريب (RMSEA) \\
\hline $1 / \Delta \Lambda$ & $\leq r$ & كاى اسكوير/درجه آزادى (x2/df) \\
\hline
\end{tabular}

نماينده مؤلفههاى توفيق طلبى، استقالالطلبى، كنترل درونسى،

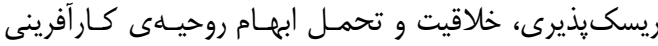

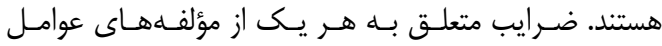

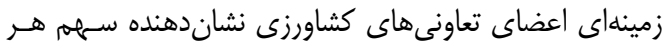

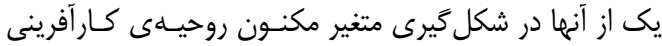

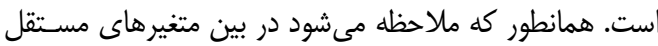

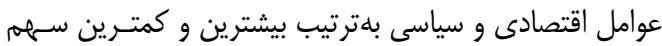

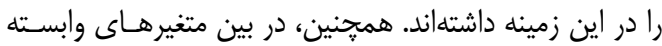

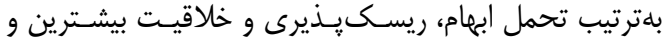

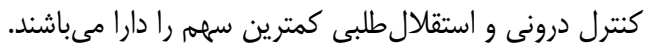

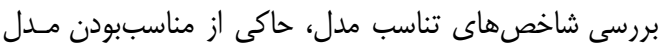

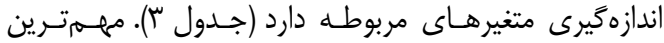
شاخص ريشه دوم برآورد واريانس خطاى تقريب مريله داست كه

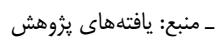

در قسمتى از تحقيق بلمنظور تحليل مسـير از مـدل معـادلات بـات

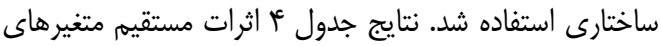

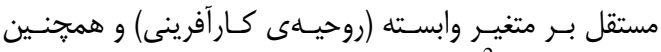

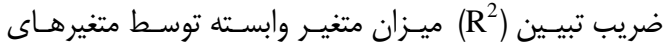

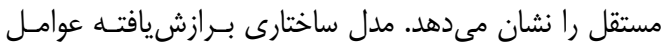

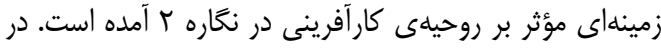

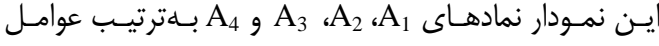

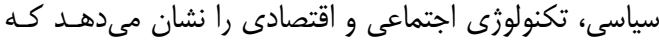

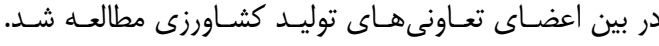

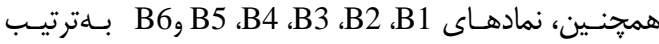




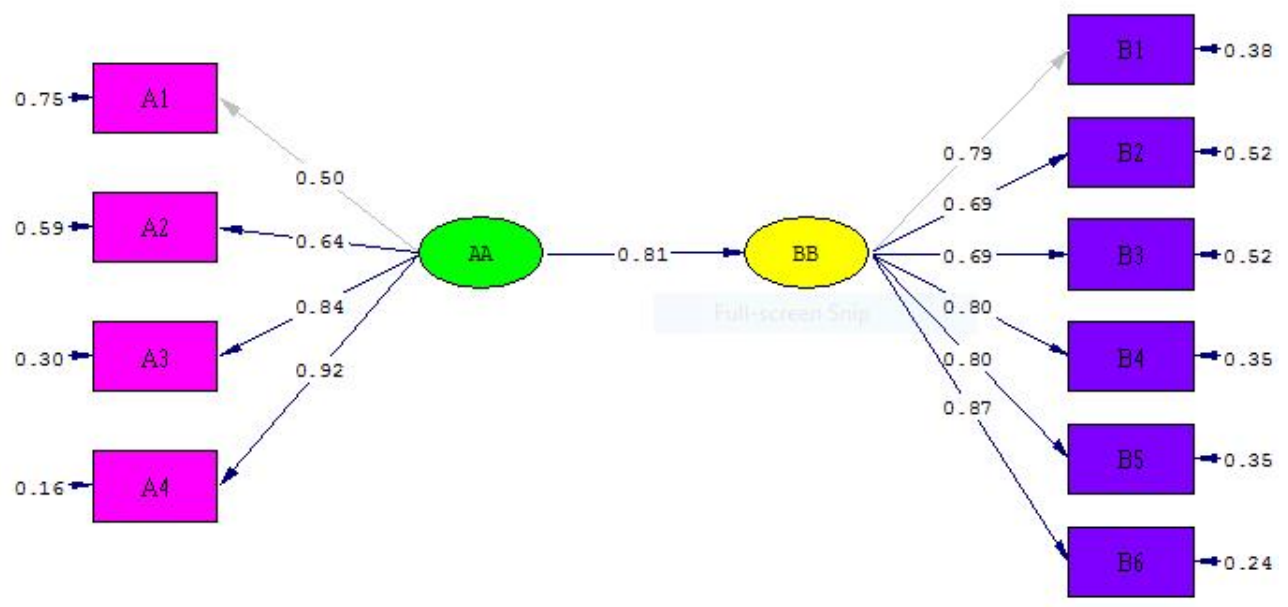

Chi-Square $=247.05, \mathrm{df}=156, \mathrm{P}-$ value $=0.00000, \mathrm{RMSEA}=0.45$

شكل r- مدل برازشيافته با نمايش بارهاى عاملى

Figure 2. The standard fitted model with the representation of factor loadings

كارآفرينى تأثير مثبت و معنى دارى داشتند. با توجـهـ بـهـ مقـــار

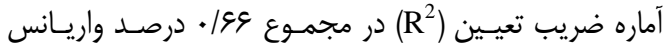

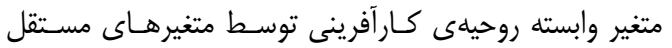
فوق تبيين مى مردد.

در جدول زير اثر عوامل زمينهاى و همجنِين ضريب تعيسين

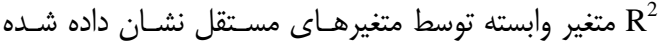

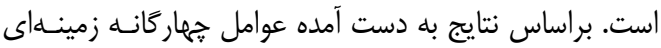

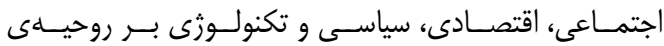
جدول f_ نتايج حاصل از آزمون فرضيههاى تحقيق

Table 4. Results of the research hypotheses' test

\begin{tabular}{|c|c|c|c|c|c|c|}
\hline $\mathrm{R}^{2}$ & آزمون فرضيه & مقدار خطاى استاندارد & $\mathrm{t}$-Values & ضريب استاندارد شده & متغير مستقل & متغير وابسته \\
\hline .199 & تأييد & $\cdot / 1 . r$ & $V / 91^{*}$ & $\cdot|\wedge|$ & عوامل زمينهاى & روحيهى كارآفرينى \\
\hline
\end{tabular}

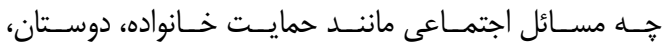

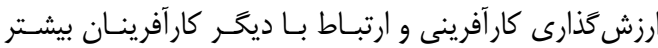

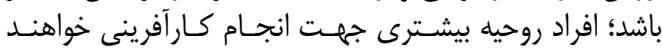

طبق نتايج بهدست آمده، عامل تكنولوزى نيز بـر روحيـهى

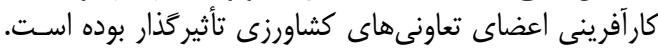

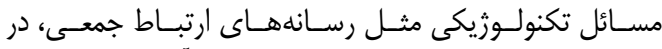

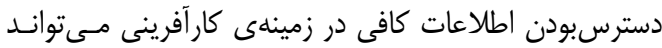

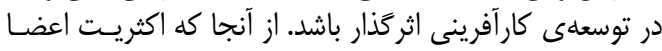

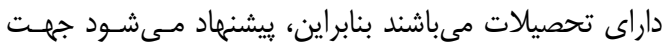

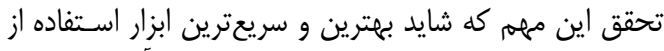

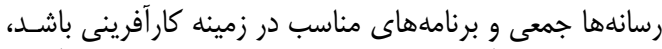

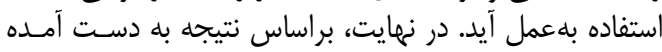

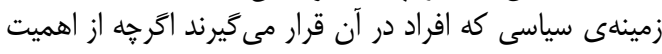

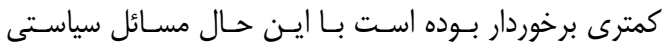

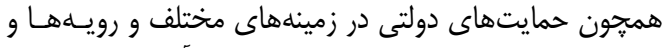

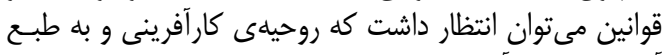

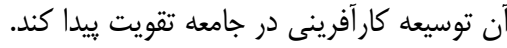

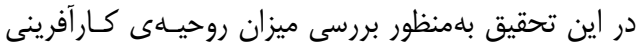

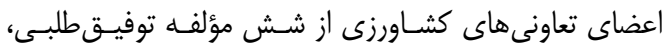

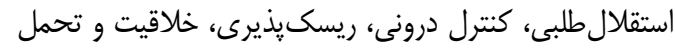

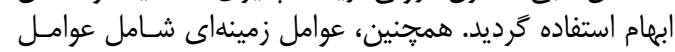

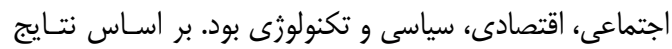

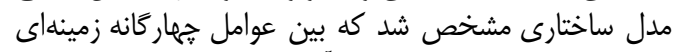

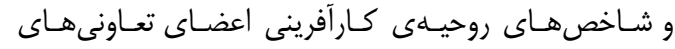

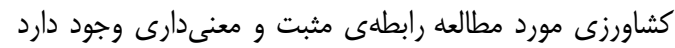

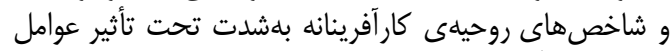

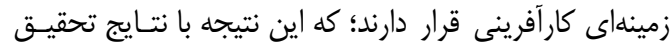

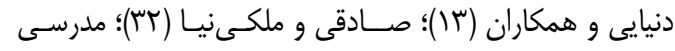
(YV)

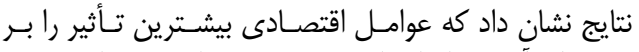

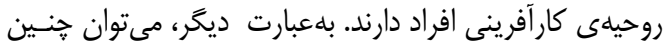

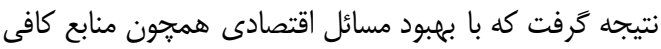

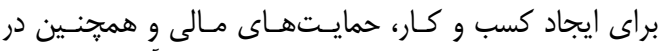

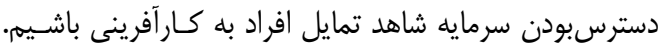

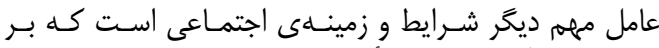

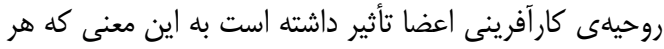


ضرورى جهت توسعهى روحيـهى كـارآفرينى مسىباشـند؛ لــذا

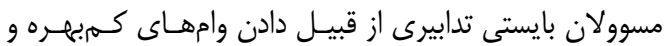

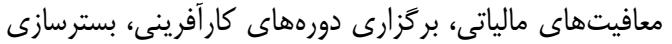

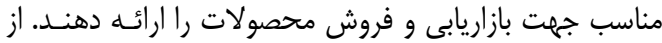

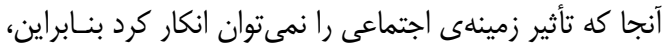

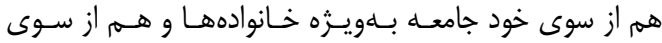

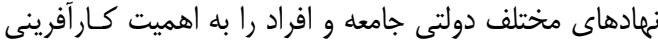
در توسعه سوق دهند.
نتايج حاكى از آن است كه عوامل زمينهاى بيشـترين تـأثير

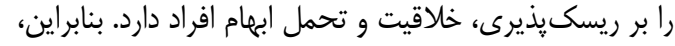

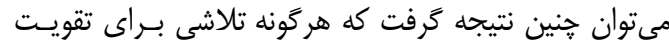

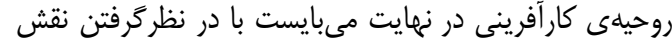

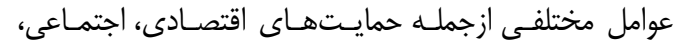

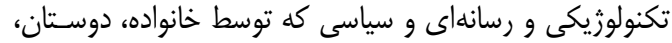

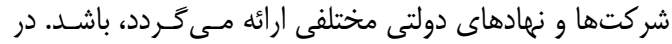

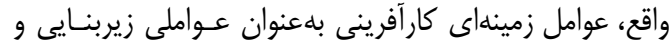

1. Ahmadi, F., R. Shafei and N.F. Mefakherinia 2012. Investigating the impact of individual an environmental factors on students' entrepreneurial behavior in Kurdistan University. Entrepreneurship Development, 15 Volume 5, Issue 1- Serial Number 15: 145-163(In Persian).

2. Abdulwahab, M.H. and R.A. Al-Damen. 2015. The impact of entrepreneurs' characteristics on small business success at medical instruments supplies organizations in Jordan. International Journal of Business and Social Science, 6(8): 164-175.

3. Alvani, S.M., S. Safari, R. Kohan Hoosh Nejad and S. Khodamoradi. 2017. Factors affecting corporate entrepreneurship: the case of food industry. Management Studies, 25(82): 1-27 (In Persian).

4. Ansari, M.T. and A. Soleimanizadeh. 2010. Investigating Environmental Factors Affecting the Development of Entrepreneurship from the Entrepreneur's Perspective. Journal of Management Research, 1(1): 87-110 (In Persian).

5. Armesh, H., C.C. Wei and G. Marthandan. 2014. Corporate entrepreneurship antecedents and firm performance in Iranian manufacturing SMEs: mediating role of corporate entrepreneurship. Journal of Basic and Applied Scientific Research, 36-51.

6. Azami, M., A. Yaghobi Farahani, E. Mousavi and A.H. Pirmoradi. 2014. Developing of organizational entrepreneurship in cooperatives. Journal of Entrepreneurship in Agriculture, 1(1): 1-18 (In Persian).

7. Bahrami, M., F. Sepaseh, M.M. Kiani, H. Yaghouti, M. Salehi and M. Khanjankhani. 2017. An investigation on entrepreneurship status of healthcare management students in Shahid Sadoughi University of medical sciences, Yazd, Iran. Management Strategies in the Health System, 2(2): 79-87 (In Persian).

8. Bashirahsan, M. and R. Ghorbaninejad. 2017. The role of entrepreneurial women in economic development (case study: Khoramrod rural district of Tuyserkan city). Geographical Territory, 14(53): 93-108 (In Persian).

9. Bird, B. and L. Schjoedt. 2017. Entrepreneurial behavior: Its nature, scope, recent research, and agenda for future research. In Revisiting the Entrepreneurial Mind (pp. 379-409). Springer, Cham

10. Busenitz, L.W. and C.M. Lau. 1996. A cross-cultural cognitive model of new venture creation. Entrepreneurship theory and practice, 20(4): 25-40.

11. Çavuş, M.F. and A. Gökçen. 2015. Psychological capital: Definition, components and effects. British Journal of Education, Society and Behavioural Science, 5(3): 244-255.

12. Deakins, D. and M. Freel. 2003. Entrepreneurship and small firms. 3thed. Berkshire: Mc Grow Hill.

13. Donyei, H., J. Yaghobi and Y. Rajaei. 2010. Factors affecting on entrepreneurship strengthening and development of agricultural cooperatives in Zanjan province. Cooperative Magazine, 3: 125-112 (In Persian).

14. Farahani, A., H. Ghasemi and N. Mohammadi. 2013. Investigating environmental factors affecting on entrepreneurial spirituality of students graduate in physical education. Applied Research in Sport Management, 2(3): 61-74 (In Persian).

15. Faraji-Sabokbar, H., S.A. Badri, H. Sajasy Ghidari, T. Sadeghloo and A. Shahdadi Khajeh Asgar. 2011. Prioritization of entrepreneurship development in rural areas by using promethean technique (case study: Suburbs of Khodabandeh district, Zanjan province). Geographic Research, 75: 68-53 (In Persian).

16. Golrd, P. 2005. Effective factors in development entrepreneurship of Iranian women's. Journal of Women's Research, 3(1): 101-123 (In Persian).

17. Guth, W.D. and A. Ginsberg. 1990. Guest editors' introduction: Corporate entrepreneurship. Strategic management journal, 5-15.

18. Haji, L., M. Chizari and Sh. Choobchian. 2016. Structural analysis of factors affecting the sustainable development of agricultural production cooperatives in rural areas Naghadeh Township. Journal of Rural Research, 7(1): 195-216 (In Persian). 
19. Hemati, M., J. Abadi and S. Islami. 2014. The relationship between organizational entrepreneurship and psychological empowerment in physical education teachers. Sport Management Studies, 26: 165180 (In Persian).

20. Histrich, R.D. and M. Drnovsek. 2002. Entrepreneurship and small business research: A European perspective. Journal of Small Business and Enterprise Development, 2: 172-222.

21. Hoshmandan-Moghaddamfard, Z., A. Razvanfar, H. Movahedmadmadi and A. Shams. 2011. Measuring the entrepreneurial spirit of students of agricultural schools in Zanjan province. Iranian Agricultural Extension and Education Journal, 7(2): 105-114 (In Persian).

22. Howard, S. 2004. Developing entrepreneurial potential in youth: The effects of entrepreneurial education and venture creation. University of South Florida Report, 3-17.

23. Kearney, C., R. Hisrich and F. Roche. 2008. A conceptual model of public sector corporate entrepreneurship. International Entrepreneurship and Management Journal, 4(3): 295-313.

24. Laspita, S., N. Breugst, S. Heblich and H. Patzelt. 2012. Intergenerational transmission of entrepreneurial intentions. Journal of Business Venturing, 27(4): 414-435.

25. Lordkipanidze, M. 2002. Enhancing entrepreneurship in rural tourism for sustainable regional development. The case of Söderslätt region. Sweden. The International Institute for Industrial Environmental Economics, IIIEE, Lund University.

26. Meysami, A.M., G. Mohammadi Elyasi, A. Mobinin Dehkordi and S.R. Heiazi. 2017. Dimensions and components of the technological entrepreneurship ecosystem in Iran. Journal of Technology Development Management, 5(3): 9-42 (In Persian).

27. Modrisi, M. 2011. Identification of institutional factors affecting on academic entrepreneurship, Tehran: Tehran University. Thesis of MSc. Faculty of Management, Entrepreneurship Department (In Persian).

28. Mokaya, S.O. 2012. Corporate entrepreneurship and organizational performance, Theoretical perspectives approaches and outcomes. International Journal of Arts and Commerce, 1(4): 133-143.

29. Pournazari, M. 2016. Analysis of factors affecting entrepreneurial behavior on agricultural entrepreneurs in Hormozgan province. Agricultural extension and education. Faculty of Agriculture, Tarbiat Modarres University, (In Persian).

30. Razaghandi, N. and K. Dorani. 2010. Identifying the individual and environmental factors affecting on success of the top entrepreneurs in Tehran province to provide a training model. Journal of Entrepreneurship Development, Second Year, 6: 55-39 (In Persian).

31. Rezaei, R. 2014. Study the role of entrepreneurship in the development of rural cooperatives. Journal of Entrepreneurship in Agriculture, 1(1): 85-102 (In Persian).

32. Sadeghi, M. and E. Malekinia. 2011. Assessing students from the entrepreneurial environment and explaining their relationship with entrepreneurial intent. Journal of Research and Planing in Higher Education, 59: 69-89 (In Persian).

33. Sobel, R.S. and K.A. King. 2008. Does school choice increase the rate of youth entrepreneurship?. Economics of Education Review, 27(4): 429-438.

34. Taghraei, M.T., M. Rezvani, M.H. Mubaraki, J.Y. Farsi and Z. Mirzaei. 2016 .Identification of environmental factors affecting cultural entrepreneurship in nomadic handicrafts. Quarterly Journal of Economics Space and Rural Development, Year 5(3): 19-36 (In Persian).

35. Toosi, R., A. Jamshidi and A. Tagdisi. 2013. Rural entrepreneurship and determining the factors affecting it (case study: villages in Minoodasht county). Journal of Rural Research and Planning, 3 (8): 2-12 (In Persian).

36. Tugraei, M.T., M. Rezvani, M.H. Mubaraki, J. Yadollahi Farsi and Z. Mirzaei. 2016. Identification of environmental factors affecting on cultural entrepreneurship in nomadic handicrafts. Journal of space economics and rural developmentt, 5(3): 19-36 (In Persian).

37. Warren, M. 2011. Econometric analysis of the impact of entrepreneurship on economic growth-Case of the Kenyan economy. Journal of Economics and Sustainable Development, 2(7): 71-88. 


\title{
Investigating the Effects of Contextual Factors on Entrepreneurial Spirit The Case of Agricultural Cooperatives in Naghadeh Township
}

\section{Latif Haji $^{1}$, Naser Valizadeh ${ }^{2}$ and Hamid Karimi ${ }^{3}$}

1- Ph.D. Student, Department of Agricultural Extension and Education, Shiraz University

2- Ph.D. Student, Department of Agricultural Extension and Education, Shiraz University (Corresponding author: n.valizadeh@shirazu.ac.ir)

3- Assistant Professor, Department of Agricultural Extension and Education, University of Zabol

Received: April 16, $2019 \quad$ Accepted: June 9, 2019

\begin{abstract}
Strengthening and paying attention to entrepreneurial spirit among the members of society are of important topics in entrepreneurship. Meeting the goals in this field requires some contextual factors that are considered as the prerequisites of ground setting for entrepreneurship. Present study aims to investigate the effects of contextual factors on the entrepreneurial spirit among members of agricultural cooperatives in Naghadeh Township. Present study is an applied research which was carried out through cross-sectional survey. Statistical population of this research was members of cooperatives $(\mathrm{N}=1170)$. Krejice and Morgan Table was used to determine the sample size $(n=290)$. A stratified random sampling method was implemented to select samples. The data collection instrument was a structured questionnaire that its validity confirmed by a panel of experts. Cronbach's alpha coefficient was calculated to test the reliability of different parts of questionnaire $(0.88 \leq \alpha \leq 0.92)$. The results of structural equation modeling showed that economic, social, technological, and political factors have positive and significant effects on entrepreneurial spirit. So that the value of the determination coefficient $\left(\mathrm{R}^{2}\right)$ could account for $0.66 \%$ of the variance of the dependent variable. According to the results, it is recommended that agricultural cooperatives be given more attention by policymakers because of their potential in entrepreneurship and improving entrepreneurial spirit.
\end{abstract}

Keywords: Agricultural Cooperatives, Contextual Factors, Entrepreneurial Spirit, Naghadeh Township 